\title{
Management of Meloidogyne incognita in Mungbean by Seed Soaking in Different Chemicals under Pot Condition
}

\author{
Mayuri Patel* and A.D. Patel \\ Department of Nematology, Anand Agricultural University, Anand-388 110, Gujarat, India \\ *Corresponding author
}

A B S T R A C T

\begin{tabular}{|l|}
\hline Ke y w o r d s \\
Chemicals, \\
$\begin{array}{l}\text { Mungbean, } M \text {. } \\
\text { incognita, } \text { Seed } \\
\text { soaking. }\end{array}$ \\
\hline Article Info \\
\hline $\begin{array}{l}\text { Accepted: } \\
\text { 10 September } 2017 \\
\text { Available Online: } \\
\text { 10 October } 2017\end{array}$ \\
\hline
\end{tabular}

\section{Introduction}

Mungbean (Vigna radiata (L) R. Wilczek) belongs to family Leguminosae and sub family Papilionaceae. It is known by many common names viz., mung, moong, mungo, goldengram, chickasawpea, oreganpea etc. In India, the name green gram is more commonly used than mungbean (Chatterjee and Randhawa, 1952). Gupta and Verma (1990) estimated avoidable losses in mungbean under field conditions due to $M$. javanica ranged from 42.1 to $93.4 \%$. The yield loss of 18 to 65 and 23 to $49 \%$ due to $M$. incognita and $M$. javanica respectively in mung bean has been reported by Sharma et al., (2000). Crop loss due to $M$. incognita in green gram has been reported as $8.90 \%$ (Khan et al., 2010). M. incognita, M. javanica, Heterodera cajani and Rotylenchulus reniformis are the nematodes attacking black gram and green gram inducing $8.90 \%$ yield loss with 162 million as a monitory loss (Jain et al., 2007).

\section{Materials and Methods}

Earthen pots of $15 \mathrm{~cm}$ diameter were washed with water and then disinfested with $4 \%$ formaldehyde (Formalin 40 EC solution). After drying, pots were filled with nematode infested soil having $1 \mathrm{~J}_{2} / \mathrm{g}$ soil $(1.5 \mathrm{~kg} / \mathrm{pot})$. Thirteen treatments including one check (Table 2) were employed in Completely Randomized Design (CRD) with four repetitions. Seeds of mungbean variety Gujarat anand mung 5 were soaked in the solution of chemicals for 6 hours. Then seeds 
were dried under shade and three seeds seeded in each pot. Upon germination, plants were thinned down to one/pot. Watering was done as and when required. Plant protection measures were adopted as per the recommendation in vogue. After 45 days of sowing, the experiment was discontinued by removing the plants from the pots and roots were washed gently under running tap water. Observations on plant height, fresh shoot and root weights were recorded. Roots were cut in to $2-3 \mathrm{~cm}$ length and $3 \mathrm{~g}$ roots were stained in $0.05 \%$ acid fuchsin in lactophenol. Then roots were washed with tap water to remove excess stain and kept overnight in lactophenol, then roots were examined for nematode population.

\section{Results and Discussion}

In order to assay the effect of chemicals for the management of Meloidogyne incognita in mungbean, seed were soaked in Acetamiprid @ $1.0 \mathrm{~g} / \mathrm{lit}$. ( $\left.\mathrm{T}_{1}\right)$, Carbosulfan @ $2.0 \mathrm{ml} / \mathrm{lit}$.
$\left(\mathrm{T}_{2}\right)$, Emamectin benzoate @ 1.0 g/lit. $\left(\mathrm{T}_{3}\right)$, Fipronil@1.0 ml/lit. ( $\left.\mathrm{T}_{4}\right)$, Imidaclopride @ 2.0 ml/lit. $\left(\mathrm{T}_{5}\right)$, Spinosad @ $1.0 \mathrm{ml} / \mathrm{lit} .\left(\mathrm{T}_{6}\right)$, Thiamethxam @1.0 g/lit. ( $\left.\mathrm{T}_{7}\right)$, Flonicamid @ 1.0 g/lit. ( $\left.\mathrm{T}_{8}\right)$, Dimethoate @ 2.0 ml/lit. ( $\left.\mathrm{T}_{9}\right)$, Chlorpyrifos@ @ $4.0 \mathrm{ml} / \mathrm{lit}$. $\left(\mathrm{T}_{10}\right)$, Methomyl 2.0 g/lit. $\left(\mathrm{T}_{11}\right)$, Triazophos @ $4.0 \mathrm{ml} / \mathrm{lit} .\left(\mathrm{T}_{12}\right)$ with a control $\left(\mathrm{T}_{13}\right)$ were tested in pot using nematode infested soil having $230 \mathrm{~J}_{2} / 100 \mathrm{cc}$ soil. Data presented in Table 1 showed nonsignificant differences for germination count.

It clearly indicates that there was no any adverse effect of chemical on seed germination. Plant height was significantly more in $T_{9}$ and $T_{3}$ followed by $T_{1}, T_{2}, T_{5}, T_{6}$, $\mathrm{T}_{7}, \mathrm{~T}_{11}$ and $\mathrm{T}_{12}$. However, all were statistically at par with each other. Minimum plant height was recorded in control $\left(\mathrm{T}_{13}\right)$ and showed non-significant difference with $\mathrm{T}_{4}, \mathrm{~T}_{5}, \mathrm{~T}_{8}, \mathrm{~T}_{6}$, $\mathrm{T}_{10}$ and $\mathrm{T}_{11}$ (Table 1). Significantly more fresh shoot weight was noticed in Emamectin benzoate @ 1.0 g/lit. $\left(\mathrm{T}_{3}\right)$ among all the treatments.

Table.1 Effect of different chemicals on plant growth characters of mungbean

\begin{tabular}{|c|c|c|c|c|}
\hline \multirow{3}{*}{ Treatment } & \multicolumn{4}{|c|}{ Plant growth parameters } \\
\hline & \multirow{2}{*}{$\begin{array}{c}\text { Germination count/ } \\
\text { pot }\end{array}$} & \multirow{2}{*}{$\begin{array}{c}\text { Plant height, } \\
\text { cm }\end{array}$} & \multicolumn{2}{|c|}{$\begin{array}{l}\text { Fresh weight, } \mathrm{g} \\
\end{array}$} \\
\hline & & & Shoot & Root \\
\hline $\mathrm{T}_{1}(\mathrm{ACET})$ & 3.00 & $17.75^{\mathrm{ab}}$ & $1.72^{\mathrm{bcd}}$ & $0.31^{\mathrm{c}}$ \\
\hline $\mathrm{T}_{2}(\mathrm{CARB})$ & 3.00 & $18.38^{\mathrm{ab}}$ & $1.78^{\mathrm{bcd}}$ & $0.38^{\mathrm{b}}$ \\
\hline $\mathrm{T}_{3}(\mathrm{EMAM})$ & 3.00 & $20.13^{\mathrm{a}}$ & $2.26^{\mathrm{a}}$ & $0.49^{\mathrm{a}}$ \\
\hline $\mathrm{T}_{4}(\mathrm{FIPR})$ & 3.00 & $16.13^{\mathrm{bc}}$ & $1.27^{\mathrm{f}}$ & $0.29^{\mathrm{c}}$ \\
\hline $\mathrm{T}_{5}(\mathrm{IMID})$ & 2.75 & $17.50^{\mathrm{abc}}$ & $1.40^{\mathrm{def}}$ & $0.29^{\mathrm{c}}$ \\
\hline $\mathrm{T}_{6}(\mathrm{SPIN})$ & 3.00 & $17.88^{\mathrm{ab}}$ & $1.77^{\mathrm{bcd}}$ & $0.32^{\mathrm{c}}$ \\
\hline $\mathrm{T}_{7}$ (THIA) & 3.00 & $18.50^{\mathrm{ab}}$ & $1.81^{\mathrm{bc}}$ & $0.41^{\mathrm{b}}$ \\
\hline $\mathrm{T}_{8}(\mathrm{FLON})$ & 3.00 & $16.00^{\mathrm{bc}}$ & $1.15^{\mathrm{f}}$ & $0.21^{\mathrm{d}}$ \\
\hline $\mathrm{T}_{9}(\mathrm{DIME})$ & 3.00 & $20.13^{\mathrm{a}}$ & $1.92^{\mathrm{ab}}$ & $0.41^{\mathrm{b}}$ \\
\hline $\mathrm{T}_{10}(\mathrm{CHLO})$ & 3.00 & $16.75^{\mathrm{bc}}$ & $1.33^{\mathrm{ef}}$ & $0.29^{\mathrm{c}}$ \\
\hline $\mathrm{T}_{11}(\mathrm{METH})$ & 2.75 & $17.50^{\mathrm{abc}}$ & $1.49^{\text {cdef }}$ & $0.30^{\mathrm{c}}$ \\
\hline $\mathrm{T}_{12}$ (TRIA) & 3.00 & $17.75^{\mathrm{ab}}$ & $1.67^{\text {bcde }}$ & $0.31^{\mathrm{c}}$ \\
\hline $\mathrm{T}_{13}(\mathrm{CON})$ & 3.00 & $14.50^{\mathrm{c}}$ & $0.70^{\mathrm{g}}$ & $0.17^{\mathrm{d}}$ \\
\hline S.Em. \pm & 0.09 & 0.97 & 0.11 & 0.01 \\
\hline C.D. 0.05 & NS & - & - & - \\
\hline C.V. \% & 6.62 & 11.03 & 14.48 & 9.75 \\
\hline
\end{tabular}

$* 0=$ Free; 5 = Maximum disease intensity. Figures indicating common letters do not differ significantly from each other at $5 \%$ level of significance according to DNMRT. 
Table.2 Effect of different chemicals on multiplication of Meloidogyne incognita on mungbean in pots

\begin{tabular}{|c|c|c|c|c|}
\hline \multirow{3}{*}{ Treatment } & \multicolumn{4}{|c|}{ Nematode population/plant } \\
\hline & \multirow{2}{*}{$\begin{array}{c}\text { RKI } \\
(\mathbf{0 - 5})^{*} \\
(\sqrt{ } \mathbf{x})\end{array}$} & \multicolumn{2}{|c|}{ Root } & \multirow{2}{*}{$\begin{array}{l}\text { Soil }(200 \mathrm{cc}) \\
\quad(\log X)\end{array}$} \\
\hline & & $\begin{array}{c}\text { Females } / 3 g \\
(\log X)\end{array}$ & $\begin{array}{c}\text { Eggs/3g } \\
(\log X)\end{array}$ & \\
\hline $\mathrm{T}_{1}(\mathrm{ACET})$ & $\begin{array}{c}2.00^{\mathrm{abcd}} \\
(4.00)\end{array}$ & $\begin{array}{c}1.27^{\mathrm{def}} \\
(127.50)\end{array}$ & $\begin{array}{c}2.85^{\text {def }} \\
(711.00)\end{array}$ & $\begin{array}{c}2.11^{\mathrm{de}} \\
(127.50)\end{array}$ \\
\hline $\mathrm{T}_{2}(\mathrm{CARB})$ & $\begin{array}{l}1.78^{\mathrm{e}} \\
(3.25)\end{array}$ & $\begin{array}{l}1.24^{\text {def }} \\
(99.75)\end{array}$ & $\begin{array}{c}2.73^{\text {def }} \\
(547.00)\end{array}$ & $\begin{array}{c}1.99^{\mathrm{f}} \\
(99.75)\end{array}$ \\
\hline $\mathrm{T}_{3}(\mathrm{EMAM})$ & $\begin{array}{l}1.57^{\mathrm{f}} \\
(2.50)\end{array}$ & $\begin{array}{c}0.99^{f} \\
(53.00)\end{array}$ & $\begin{array}{c}2.30^{\mathrm{i}} \\
(212.00)\end{array}$ & $\begin{array}{c}1.72^{\mathrm{g}} \\
(53.00)\end{array}$ \\
\hline $\mathrm{T}_{4}(\mathrm{FIPR})$ & $\begin{array}{l}2.05^{\mathrm{ab}} \\
(4.25)\end{array}$ & $\begin{array}{c}1.65^{\mathrm{bc}} \\
(181.50)\end{array}$ & $\begin{array}{c}3.20^{\mathrm{b}} \\
(1679.50)\end{array}$ & $\begin{array}{c}2.26^{\mathrm{bc}} \\
(181.50)\end{array}$ \\
\hline $\mathrm{T}_{5}(\mathrm{IMID})$ & $\begin{array}{c}2.00^{\mathrm{abcd}} \\
(4.00)\end{array}$ & $\begin{array}{c}1.59^{\mathrm{bc}} \\
(166.50)\end{array}$ & $\begin{array}{c}2.95^{\mathrm{cd}} \\
(898.75)\end{array}$ & $\begin{array}{c}2.21^{\mathrm{bc}} \\
(166.50)\end{array}$ \\
\hline $\mathrm{T}_{6}(\mathrm{SPIN})$ & $\begin{array}{l}1.79^{\text {cde }} \\
(3.25)\end{array}$ & $\begin{array}{c}1.26^{\mathrm{def}} \\
(108.25)\end{array}$ & $\begin{array}{c}2.80^{\mathrm{ef}} \\
(637.25)\end{array}$ & $\begin{array}{c}2.03^{\mathrm{ef}} \\
(108.25)\end{array}$ \\
\hline $\mathrm{T}_{7}(\mathrm{THIA})$ & $\begin{array}{l}1.73^{\text {ef }} \\
(3.00)\end{array}$ & $\begin{array}{l}1.22^{\text {def }} \\
(99.50)\end{array}$ & $\begin{array}{c}2.60^{\mathrm{gh}} \\
(410.25)\end{array}$ & $\begin{array}{c}1.99^{\mathrm{f}} \\
(99.50)\end{array}$ \\
\hline $\mathrm{T}_{8}(\mathrm{FLON})$ & $\begin{array}{l}2.05^{\mathrm{ab}} \\
(4.25)\end{array}$ & $\begin{array}{c}1.78^{\mathrm{ab}} \\
(210.75)\end{array}$ & $\begin{array}{c}3.38^{\mathrm{a}} \\
(2451.75)\end{array}$ & $\begin{array}{c}2.32^{\mathrm{b}} \\
(210.75)\end{array}$ \\
\hline $\mathrm{T}_{9}(\mathrm{DIME})$ & $\begin{array}{l}1.79^{\text {cde }} \\
(3.25)\end{array}$ & $\begin{array}{c}1.16^{\mathrm{ef}} \\
(89.00)\end{array}$ & $\begin{array}{c}2.48^{\mathrm{h}} \\
(311.25)\end{array}$ & $\begin{array}{c}1.94^{\mathrm{f}} \\
(89.00)\end{array}$ \\
\hline $\mathrm{T}_{10}(\mathrm{CHLO})$ & $\begin{array}{l}2.05^{\mathrm{ab}} \\
(4.25)\end{array}$ & $\begin{array}{c}1.62^{\mathrm{bc}} \\
(168.25)\end{array}$ & $\begin{array}{c}3.08^{\mathrm{bc}} \\
(1267.50)\end{array}$ & $\begin{array}{c}2.22^{\mathrm{bc}} \\
(168.25)\end{array}$ \\
\hline $\mathrm{T}_{11}(\mathrm{METH})$ & $\begin{array}{c}2.00^{\mathrm{abcd}} \\
(4.00)\end{array}$ & $1.52^{\mathrm{bcd}}(160.00)$ & $\begin{array}{c}2.91^{\mathrm{de}} \\
(815.50)\end{array}$ & $\begin{array}{c}2.19^{\text {cd }} \\
(160.00)\end{array}$ \\
\hline $\mathrm{T}_{12}$ (TRIA) & $\begin{array}{c}1.93^{\text {bcde }} \\
(3.75)\end{array}$ & $\begin{array}{c}1.40^{\text {cde }} \\
(146.25)\end{array}$ & $\begin{array}{c}2.89^{\mathrm{de}} \\
(792.00)\end{array}$ & $\begin{array}{c}2.16^{\mathrm{cd}} \\
(146.25)\end{array}$ \\
\hline $\mathrm{T}_{13}(\mathrm{CON})$ & $\begin{array}{l}2.17^{\mathrm{a}} \\
(4.75)\end{array}$ & $\begin{array}{c}2.01^{\mathrm{a}} \\
(362.50)\end{array}$ & $\begin{array}{c}3.45^{\mathrm{a}} \\
(2886.25)\end{array}$ & $\begin{array}{c}2.55^{\mathrm{a}} \\
(362.50)\end{array}$ \\
\hline S.Em. \pm & 0.09 & 0.09 & 0.04 & 0.03 \\
\hline C.V. \% & 6.62 & 12.71 & 2.93 & 3.09 \\
\hline
\end{tabular}

$* 0=$ Free 5 = Maximum disease intensity.

Figures in parentheses are re-transformed values of $\sqrt{x}$ and $\log X$.

Figures indicating common letters do not differ significantly from each other at $5 \%$ level of significance according to DNMRT. 
Plate.1 Effect of seed soaking of chemicals on management of $M$. incognita
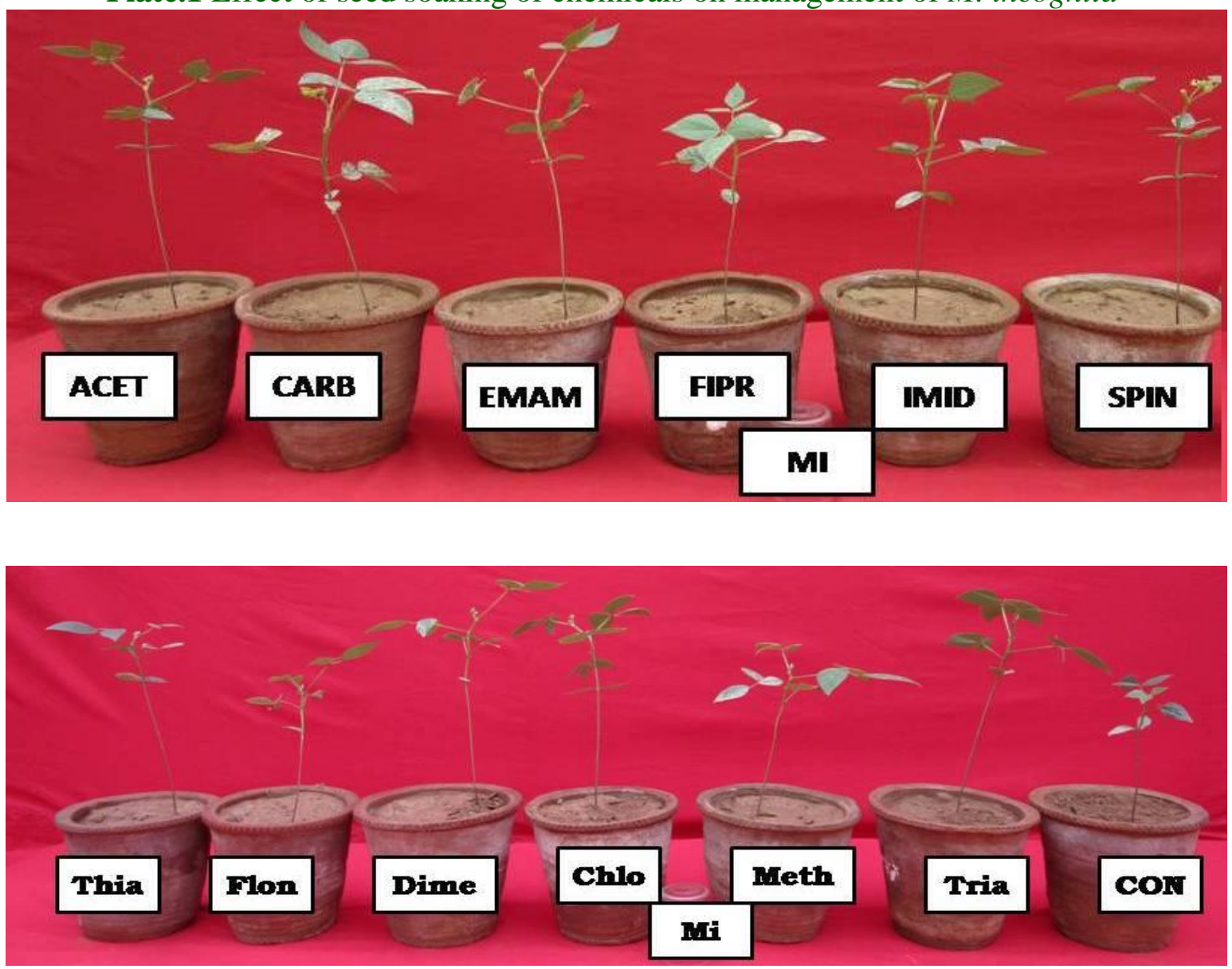

(A) SHOOT

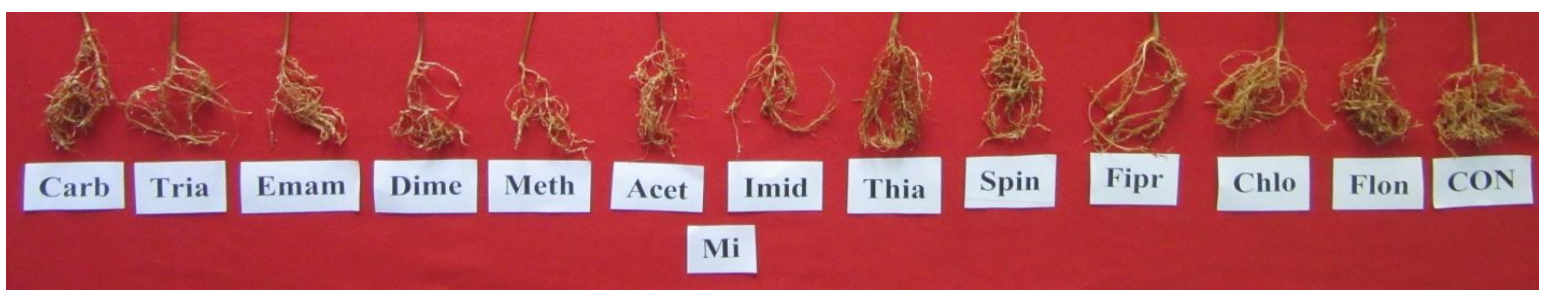

(B) ROOT

Treatment of Dimethoate @ $2.0 \mathrm{ml} / \mathrm{lit} .\left(\mathrm{T}_{9}\right)$ found next best treatment and remain statistically at par with Emamectin benzoate @ $1.0 \mathrm{~g} / \mathrm{lit}$. $\left(\mathrm{T}_{3}\right)$. Control had significantly lowest fresh shoot weight. Rest of the treatment has mediocre effect for fresh shoot weight (Table 1, Plate 1). Maximum fresh root weight was recorded in Emamectin benzoate @ 1.0 g/lit. $\left(\mathrm{T}_{3}\right)$ and it differ significantly from rest of the treatments. Treatments, $\mathrm{T}_{2}, \mathrm{~T}_{7}$ and $\mathrm{T}_{9}$ were next best treatments and remain at par with each other and differ significantly with Emamectin benzoate@1.0 g/lit. $\left(\mathrm{T}_{3}\right)$. Control $\left(\mathrm{T}_{13}\right)$ has significantly low fresh root weight. It was statistically at par with Flonicamid @ 1.0 $\mathrm{g} /$ lit. $\left(\mathrm{T}_{8}\right)$. Rest of the treatment has midiocare effect (Table 1, Plate 1). Root knot index was 
significantly higher in the $\mathrm{T}_{13}$ (control). However, it was statistically at par with the treatment $\mathrm{T}_{1}, \mathrm{~T}_{4}, \mathrm{~T}_{5}, \mathrm{~T}_{8}, \mathrm{~T}_{10}, \mathrm{~T}_{11}$ and $\mathrm{T}_{12}$. RKI was significantly less in the treatment of Emamectin benzoate @ 1.0 g/lit. $\left(\mathrm{T}_{3}\right)$ as compared to rest of the treatments (Table 2). Treatment $\mathrm{T}_{3}$ found significantly superior over other treatment having least no. of females/plant, but it did not differ with the treatment of $\mathrm{T}_{1}, \mathrm{~T}_{2}, \mathrm{~T}_{6}, \mathrm{~T}_{7}$ and $\mathrm{T}_{9}$. Maximum females were recorded in $\mathrm{T}_{13}$ which is statistically at par with $\mathrm{T}_{8}$. In cause of eggs, treatment $\mathrm{T}_{3}$ found most effective which has significantly less eggs over other treatments. Maximum no. of eggs was recorded in treatment $\mathrm{T}_{13}$ which is statistically at par with $\mathrm{T}_{8}$. Rest of the treatments has mediocre effect. In case of soil nematode population treatment $\mathrm{T}_{3}$ turn out significantly better which has lowest nematode population. Treatment $T_{2}$, $\mathrm{T}_{6}, \mathrm{~T}_{7}$ and $\mathrm{T}_{9}$ were statistically at par with each other and closely followed to $T_{3}$. Significantly higher nematode population was rescored in $\mathrm{T}_{13}$ (Table 2 ).

\section{References}

Chatterjee, D., and Randhawa, G. S. 1952. Standardized Names of Cultivated Plants in India. Indian J. Horticulture. 9 (2): 24-36.
Gupta, D. C., and Verma, K. K. 1990. Studies on avoidable losses in mung bean (Vigna radiata) due to root-knot nematode, Meloidogyne javanica and its control under field conditions. Indian J. Nematol. 20 (2): 148-151.

Jain, R. K., Mathur, K. N. and Singh, R. V. 2007. Estimation of Losses due to Plant Parasitic Nematodes on different Crops in India. Indian J. Nematol. 37 (2): 219221.

Khan, M. R., Jain, R. K., Singh, R. V. and Pramanik, A. 2010. Economically Important Plant Parasitic Nematodes Distribution Atlas. Directorate of Information and Publications of Agricuture, New Delhi. pp: 10.

Sasser, J. N., and Freckman, D. W. 1987. A world prospective on nematology: the role of the society, In: Vistas on Nematology, Edited by J.A. Veech and D.W. Dickson. Society of Nematologists, Hyattsville, MD. Pp: 714

Sharma, S. B., Sharma, H. K. and Pankaj 2000. Nematodes problem in India In: Crop pest and Disease managementchallenges for the millennium (Edited by D. Prasad and S.N. Puri), Joyti Publishers, New Delhi: 267-275.

\section{How to cite this article:}

Mayuri Patel and Patel, A.D. 2017. Management of Meloidogyne incognita in Mungbean by Seed Soaking in Different Chemicals under Pot Condition. Int.J.Curr.Microbiol.App.Sci. 6(10): 1080-1084. doi: https://doi.org/10.20546/ijcmas.2017.610.131 